\section{The effects of prebiotic, probiotic and synbiotic diets containing Bacillus coagulans and inulin on serum lipid profile in the rat}

\author{
Khadijeh Abhari, 1 \\ Seyed Shahram Shekarforoush, 1 \\ Saeed Hosseinzadeh,1 Saeed Nazifi,2 \\ Javad Sajedianfard 3 \\ 1Department of Food Hygiene and Public \\ Health, 2Department of Clinical Studies, \\ 3Department of Basic Sciences, School \\ of Veterinary Medicine, Shiraz University, \\ Iran
}

\section{Abstract}

An in vivo trial was conducted to evaluate the effects of Bacillus coagulans, and inulin, either separately or in combination, on lipid profile using a rat model. Thirty-two male Wistar rats were randomly divided into four groups $(\mathrm{n}=8)$ and fed as follows: standard diet (control), standard diet with 5\% w/w long chain inulin (prebiotic), standard diet with $10^{9}$ spores/day spores of $B$. coagulans by orogastric gavage (probiotic), and standard diet with $5 \%$ w/w long chain inulin and $10^{9}$ spores/day of $B$. coagulans (synbiotic). Rats were fed for 30 days. Serum samples were collected 10, 20 and 30 days following onset of treatment. Total, HDL and LDL cholesterol and triglycerides concentrations were analyzed. Results of this study showed that inulin potentially affected the lipid profile. An obvious decrease in serum total cholesterol and LDL-cholesterol of rats fed with inulin in symbiotic and prebiotic groups was seen in all sampling days. Inulin fed rats also demonstrated higher levels of HDL-cholesterol concentration; however this value in probiotic and control fed rats remains without significant change. According to the results of this study, $B$. coagulans did not contribute to any lipid profile changes after 30 days. Thus, further in vitro investigations on the characteristic of these bacteria could be useful to gain insights into understanding the treatment of probiotics in order to achieve the maximum beneficial effect.

\section{Introduction}

WHO has predicted that by 2030 , cardiovascular diseases will be the most important cause of death, affecting approximately 23.6 million people around the world. ${ }^{1}$ People affected with hypercholesterolemia are at a three times higher risk of heart attack compared to those who have normal blood lipid profiles. ${ }^{2}$ Pharmacological agents are able to reduce cholesterol levels effectively; however, they are expensive and the undesirable side effects have caused concerns about their therapeutic use. Therefore many investigations have been done to evaluate new approaches toward the identification of other dietary means of reducing blood cholesterol levels. These include dietary supplementation of probiotics and/or prebiotics. Probiotics are defined as living microbial supplements that beneficially affect the host animals by improving its intestinal microbial balances. ${ }^{3}$ Prebiotics are indigestible fermented food substrates that selectively stimulate the growth, composition and activity of microflora in gastrointestinal tract and thus improve hosts' health and well-being. ${ }^{4}$ When probiotics and prebiotics are used in combination they are known as synbiotics.

Micro-organisms used as a probiotic for human mainly belong to the Lactobacillus and Bacillus spp. Bacillus probiotics differ in many characteristics from those based on Lactobacillus spp. While Lactobacillus represents a normal resident gastrointestinal tract (GI) microflora of humans, Bacillus belongs only to the transitory GI bacteria. ${ }^{5}$ Most Lactobacillus probiotics are inactivated by bile and low gastric $\mathrm{pH}$, whereas members of genus Bacillus are endospore forming bacteria that make it extremely heat-stable and resistant to adverse GI tract conditions and when germinate in GI tract, cause positive effects for the host. B. coagulans (reported incorrectly as Lactobacillus sporogenes $)^{6}$ is a shelf stable bacteria that secretes L (+) lactic acid, shortchain fatty acids such as butyric acid and a bacteriocin called Coagulin, which has activity against a broad spectrum of enteric microbes.

Although many controversial studies have demonstrated the cholesterol-lowering effects of probiotics, prebiotics and synbiotics in animals and humans, there is also limited information on cholesterol-lowering effects of $B$. coagulans spores. Studies on the effects of $B$. coagulans on lipid profile have been limited to those who investigated the influence of administration of $B$. coagulans capsules (each containing 360 million spores) per day in hyperlipidemic patients for three months, they reported total serum cholesterol, LDL cholesterol and total cholesterol to HDL cholesterol, and LDL-cholesterol to HDL-cholesterol ratios were reduced significantly. They also found HDL-cholesterol was marginally increased. ${ }^{7}$ Panda et al.$^{8}$ also reported this probiotic is able to reduce total cholesterol, VLDL and triglycerides in broiler chickens.

Considering our previous findings indicated significant changes in rat GI tract microbiota
Correspondence: Saeed Nazifi, Department of Clinical Studies, School of Veterinary Medicine, Shiraz University, Shiraz, P.0. Box: 1731-71345, Iran.

Tel.: +98-.711.2286940 - Fax: +98.711 .2286950$

E-mail: nazifi@shirazu.ac.ir

Key words: Bacillus coagulans; inulin; lipid profile; synbiotic diet; rat.

Acknowledgement: this research was financially supported by "Natural Antimicrobials Centre of Excellence (NACE)" which is gratefully acknowledged.

Contributions: the authors contributed equally.

Conflict of interest: the authors declare no potential conflict of interest.

Received for publication: 21 March 2015.

Accepted for publication: 29 April 2015

This work is licensed under a Creative Commons Attribution NonCommercial 3.0 License (CC BYNC 3.0).

(C) Copyright K. Abhari et al., 2015

Licensee PAGEPress srl, Italy

Veterinary Science Development 2015; 5:5919

doi:10.4081/vsd.2015.5919

following administration of $B$. coagulans and inulin, ${ }^{9}$ this study was conducted to evaluate the in vivo effects of $B$. coagulans and inulin, separately and in combination on lipid profile using a rat model.

\section{Materials and Methods}

\section{Preparation of spore suspension of probiotic bacteria}

Lyophilized probiotic B. coagulans were donated by the Pardis Roshd Mehregan Company, Iran. It was grown aerobically in Nutrient Yeast extract Salt Medium (NYSM) agar 10 at $37^{\circ} \mathrm{C}$ for $24 \mathrm{~h}$. A single colony from the NYSM plate was inoculated into $500 \mathrm{~mL}$ of NYSM broth and incubated at $37^{\circ} \mathrm{C}$ with shaking at $250 \mathrm{rpm}$ for $48 \mathrm{~h}$. The bacterial suspension was pelleted three times by centrifugation at $3000 \times \mathrm{g}$ for $20 \mathrm{~min}$, and washed with sterile normal saline. Final pellet was re-suspended in $100 \mathrm{~mL}$ sterile normal saline. To determine the spore per ml of suspension, the solution was heated at $80^{\circ} \mathrm{C}$ for $15 \mathrm{~min}$ to kill the vegetative cells before appropriate serial dilution and plating in NYSM agar. Finally, the spore suspension was prepared at a concentration of $1 \times 10^{9} \mathrm{spore} / \mathrm{mL}$ in sterile saline and kept in the refrigerator until use. 


\section{Animals and diets}

Thirty-two male Wistar rats $(200 \pm 8.4 \mathrm{~g})$ were provided by the Animal Centre of Razi Research Institute, Shiraz, Iran. Animals were randomly assigned to four dietary groups ( $n=8 /$ group) and housed in groups of six rats per cage in a temperature controlled environment $\left(22 \pm 2^{\circ} \mathrm{C}\right)$ with $55 \pm 10 \%$ relative humidity and controlled lighting (12 h light/dark cycle).

Rats were randomly divided into 4 groups and fed as follows: i) standard diet (control), ii) standard diet supplemented with $5 \% \mathrm{w} / \mathrm{w}$ long chain inulin (Sensus, Netherlands) (prebiotic), iii) standard diet with $10^{9}$ spores/day B. coagulans (gavage $1 \mathrm{ml}$ of prepared spore suspension using a blunt ended needle) (probiotic), and iv) standard diet supplied with 5\% w/w long chain inulin and $10^{9}$ spores/day $B$. coagulans (synbiotic). The standard pellet feedstuff contained $14.5 \%$ protein, $4.7 \%$ ash,
$51.2 \%$ starch, $4.3 \%$ sugar and $4 \%$ fat $(3.2$ $\mathrm{kcal} / \mathrm{g}$ ). Regarding micronutrients, the feedstuff contained $0.72 \%$ calcium, $0.6 \%$ phosphorus, $0.23 \%$ magnesium and $0.25 \%$ chloride among others. The inulin content in the rat diet was calculated based on food intake. The food intake of each rat with mean of $200 \mathrm{~g}$ body weight is $10 \mathrm{~g} /$ day, it means each rat received $0.5 \mathrm{~g}$ inulin/day. Food and distilled water were provided ad libitum.

To assimilate the experimental conditions, the control and prebiotic group were gavaged with $1 \mathrm{~mL}$ of sterile normal saline once a day.

\section{Animal ethics}

This experiment was accomplished under the approval of the state committee on animal ethics, Shiraz University, Shiraz, Iran. Also, the recommendations of European Council Directive (86/609/EC) of November 24, 1986 were followed, regarding the standards in the protection of animals used for experimental purposes.

\section{Experimental design and sampling}

All animals were acclimatized for 2 weeks before the experimental session. Rats were fed the diets for 30 days. On day 10, 20 and 30 of trial, animals were anesthetized with Diethyl ether and $3 \mathrm{~mL}$ blood samples were collected from each animal through heart puncture and left to stand for $30 \mathrm{~min}$ at room temperature $\left(20^{\circ} \mathrm{C}\right)$ for coagulation before being centrifuged at $750 \mathrm{~g}$ for 15 minutes. Serum samples were stored at $-20^{\circ} \mathrm{C}$ until analysis.

\section{Lipid components analysis}

The analysis of the serum for total cholesterol was done using a commercial kit (Ziest Chem Diagnostics, Tehran, Iran) by a modified

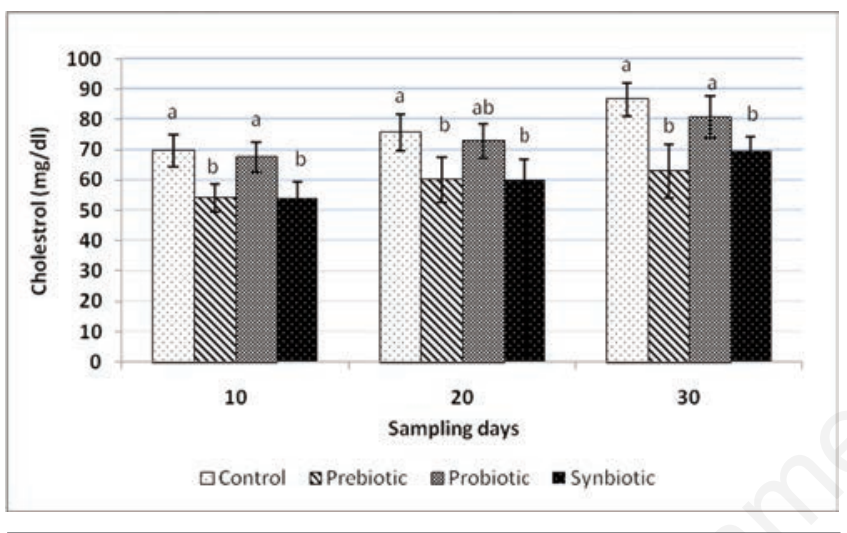

Figure 1. Effect of oral administration of Bacillus coagulans (probiotic diet) and inulin (prebiotic diet) separately and in combination (synbiotic diet) on serum total cholesterol levels in male Wistar rats. Values are expressed as mean for 8 animals. Bars represent standard deviation values. The different letters in the same sampling day indicate significant differences $(\mathbf{P}<0.05)$

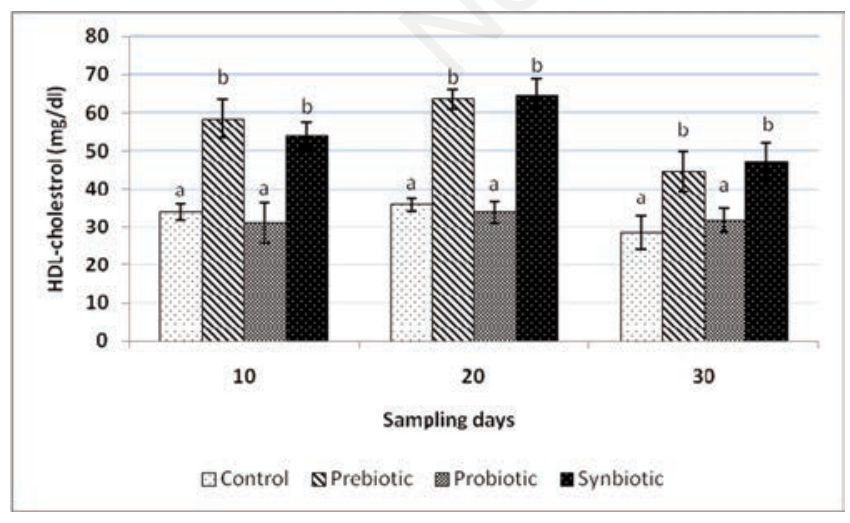

Figure 2. Effect of oral administration of Bacillus coagulans (probiotic diet) and inulin (prebiotic diet) separately and in combination (synbiotic diet) on serum triglycerides levels in male Wistar rats. Values are expressed as mean for 8 animals. Bars represent standard deviation values. The different letters in the same sampling day indicate significant differences $(P<0.05)$.

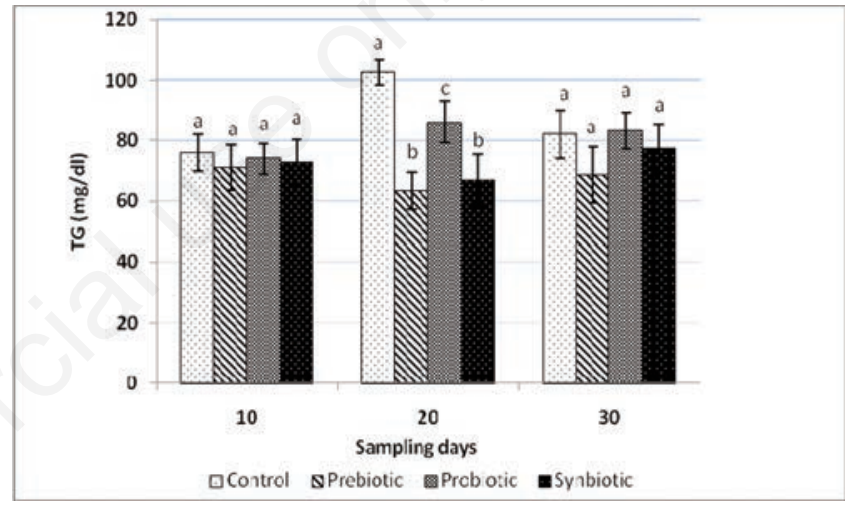

Figure 3. Effect of oral administration of Bacillus coagulans (probiotic diet) and inulin (prebiotic diet) separately and in combination (synbiotic diet) on serum HDL-cholesterol levels in male Wistar rats. Values are expressed as mean for 8 animals. Bars represent standard deviation values. The different letters in the same sampling day indicate significant differences $(P<0.05)$.

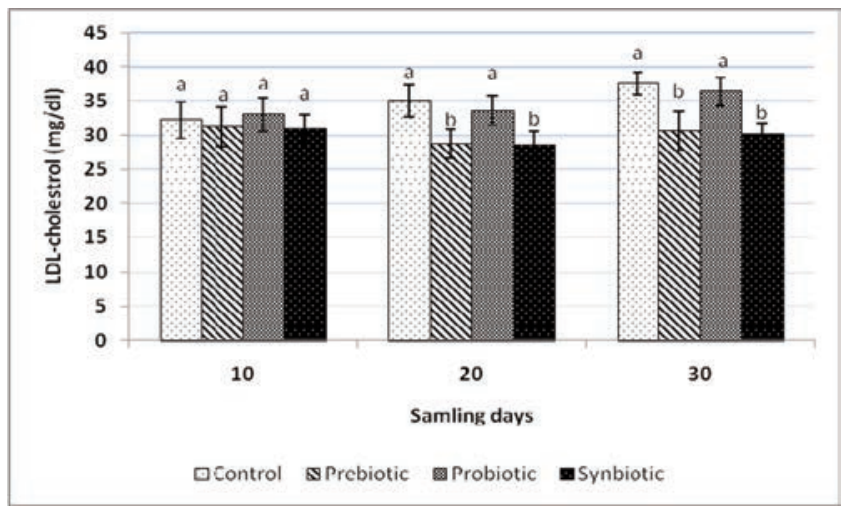

Figure 4. Effect of oral administration of Bacillus coagulans (probiotic diet) and inulin (prebiotic diet) separately and in combination (synbiotic diet) on serum LDL-cholesterol levels in male Wistar rats. Values are expressed as mean for 8 animals. Bars represent standard deviation values. The different letters in the same sampling day indicate significant differences $(P<0.05)$. 
Abell-Kendall/Levey-Brodie (A-K) method and the measurement of serum triglyceride was accomplished based on the enzymatic procedure by a commercial kit (Ziest Chem Diagnostics, Tehran, Iran).

Lipoproteins including HDL-cholesterol (mg/dL), and LDL-cholesterol (mg/dL) were analyzed by quantitative enzymatic colorimetric method using test kits supplied by STANBIO Laboratories, Boerne, TX, USA. All reactions were measured using Digital UV/VIS Spectrophotometer (CE 292, series 2, Cecil Instruments, Cambridge, England).

\section{Statistical analysis}

In order to determine the difference among treatments, an Analysis of Variance (ANOVA) was used and when differences were detected, a Duncan's multiple comparison test was used to differentiate the treatment means. The analysis was carried out using SPSS (version 19 , SPSS Inc) at a significance level of 0.05 .

\section{Results}

The effect of different diets supplementation on serum total cholesterol is shown in Figure 1. Supplementation with inulin caused significant decrease in serum total cholesterol of rats, on prebiotic and synbiotic diets after 10, 20 and 30 days of treatment initiation.

On day 10 , there were no significant differences in triglycerides values between treatments and control (Figure 2). By day 20, serum triglycerides concentrations in control, probiotic, prebiotic and symbiotic groups were 102.6, 86.1, 63.2 and $66.9 \mathrm{mg} / \mathrm{dL}$, respectively, which shows inulin has a potential role in triglycerides decrease $(\mathrm{P}<0.001)$. On day 30 , lower serum triglycerides $(\mathrm{P}=0.23)$ was seen in prebiotic and synbiotic groups compared to probiotic and control but it was not significant.

On day 10, 20 and 30 of trial HDL-cholesterol level increased significantly $(\mathrm{P}<0.001)$ in serum of rats fed with inulin, in prebiotic and symbiotic groups, while rats fed by only $B$. coagulans remained without significant change (Figure 3).

Changes in LDL-cholesterol level during 30 days treatment with experimental diets are shown in Figure 4. Treatment with different diets appeared to have no effect on the LDL cholesterol concentration in rats by day 10 . However, supplementation with prebiotic and synbiotic diets significantly lowered LDL-cholesterol concentration over 20 and 30 days; rats on the probiotic and control diets showed increased in LDL-cholesterol values.

\section{Discussion}

This feeding trial was conducted to investigate the effect of $B$. coagulans and inulin, separately and in combination on serum lipid profile using rats as a model. Administration of inulin in rats on prebiotic and synbiotic diets showed significantly $(\mathrm{P}<0.05)$ lower total cholesterol level compared to probiotic and control rats in day 10, 20 and 30 . Significant changes in triglyceride concentration are limited to day 20 , when rats on prebiotic and symbiotic diets showed lower levels of triglyceride concentrations, while on day 10 and 30 no significant differences between groups were observed.

According to the results of this study, inulin played an important role in changing lipid profile effectively. An obvious decrease in the serum total cholesterol and LDL-cholesterol of rats fed with inulin in synbiotic and prebiotic groups was seen in all sampling days. Inulin fed rats also demonstrated higher levels of HDL-cholesterol concentration; however this value in probiotic and control fed rats showed no significant change.

There are two suggested mechanisms that have been attributed to hypocholesrolemic effect of prebiotics such as inulin; decreased cholesterol absorption by enhancing cholesterol excretion via feces and the selective fermentation by intestinal bacterial microflora causing production of short-chain fatty acids (SCFAs). ${ }^{11}$

Results of this study concerning hypocholesterolemic effect of inulin are in agreement with several studies conducted in vivo trials. Kim and Shin ${ }^{12}$ reported administration of inulin in hypercholesterolemic rats for 4weeks decreased serum LDL-cholesterol with increased serum HDL-cholesterol levels $(\mathrm{P}<0.05)$ compared to the control.12 Causey et al. ${ }^{13}$ showed consuming $20 \mathrm{~g} /$ day inulin for 3 weeks caused significant decrease in serum triglycerides in twelve subjects. ${ }^{13}$ Similarly, in another study involving eight healthy volunteers with a daily consumption of $10 \mathrm{~g}$ of inulin for 3 weeks, significant decrease in plasma triacylglycerides concentrations compared to the placebo was observed.14 In another study by Brighenti et al., 15 significant $(\mathrm{P}<0.05)$ reduction in plasma total cholesterol and triacylglycerols of twelve healthy rats was seen following 12-week consumption of $50 \mathrm{~g}$ of a rice-based ready-to-eat cereal containing $18 \%$ inulin. 15 According to the results of this study, B. coagulans did not contribute to any lipid profile changes after 30 days. Although many studies have demonstrated cholesterol-lowering effects of probiotics in both animals and humans, debatable results have also been reported concerning inability of a particular strain of probiotic bacteria to improve lipid profile. A study by Hatakka et al. ${ }^{16}$ reported that administration of $L$. rhamnosus LC705 (1010 CFU/g per capsule; two capsules daily) did not influence blood lipid profiles in thirty-eight men after a 4-week treatment. ${ }^{16}$ Simons et al. ${ }^{17}$ and Lewis and Burmeister ${ }^{18}$ also refuted effect of $L$. fermentum, $\left(2 \times 10^{9} \mathrm{CFU}\right.$ per capsule; four capsules daily) and $L$. acidophilus on human lipid profiles, respectively.

Several possible mechanisms for hypocholesterolemic effects of probiotics are: incorporation of cholesterol into the cellular membranes by growing cells and deconjugation of bile via bile salt hydrolase. Once deconjugated, bile acids are less soluble and absorbed by the intestines, leading to increasing their rates of excretion in the feces. Cholesterol is used to synthesize new bile acids in a homeostatic response, resulting in lowering of serum cholesterol. ${ }^{19-21}$

Ooi and Liong 20 in a review article attributed these controversial findings to various factors such as different strains of probiotics, administration dosage, analytical accuracy of lipid analyses, duration of treatment period, clinical characteristic of subjects, inadequate sample sizes, and lack of suitable controls or placebo groups. ${ }^{2}$

Considering the mentioned reasons hypothesized by Ooi and Liong, ${ }^{20}$ normolipidemic condition of used rat model in the current study may be a reason for failure of these probiotic bacteria in changing lipid profile. ${ }^{20}$ In addition to all of the above, the feeding period of 30 days may not be sufficient to observe a significant change in lipid profile.

However, the strains of bacteria used as a cholesterol lowering agent must be bile tolerant, have the ability to deconjugate bile acids, and bind cholesterol. In addition, the ability of particular strain of probiotic bacteria to attach permanently to the gut wall and hence continuous supply might be necessary to exert the effects. ${ }^{21,22}$ The results of our previous study indicated that these bacteria are not able to colonize the intestine and are quickly eliminated in feces. ${ }^{9}$ As such, daily consumption of probiotic products is necessary for any longterm effect on metabolism.

\section{Conclusions}

Prebiotic inulin significantly reduced the serum total cholesterol, LDL-cholesterol and increased the HDL cholesterol of rats during the treatment period. But no influence of probiotic $B$. coagulans on lipid profile was observed. In order to justify the cholesterollowering effect of $B$. coagulans, in vitro studies proposed mechanisms of action are necessary. Thus, further investigations on the characteristic of these bacteria could be useful to gain insights into understanding the treatment of 
probiotics in order to achieve the maximum beneficial effect.

\section{References}

1. WHO. Cardiovascular disease; Fact sheet N 317, Geneva, Switzerland, September, 2009. Available from: http://www.who.int/mediacentre/factsheets/fs317/en/print.html. Accessed on 19 May 2010.

2. WHO. Diet, nutrition and prevention of chronic diseases; Report of a joint WHO/FAO expert consultation. Geneva, Switzerland, 2003. Available from: http://www.who.int/dietphysicalactivity/pu blications/trs916/en/

3 . FAO, WHO. Health and nutritional properties of probiotics in food including powder milk with live lactic acid bacteria. Report of a joint FAO/WHO expert consultation on evaluation of health and nutritional properties of probiotics in food including powder milk with live lactic acid bacteria. Cordoba, Argentina, 1-4 October, 2001. Available from: ftp://ftp.fao.org/docrep /fao/009/a0512e/a0512e00.pdf

4. Roberfroid M. Prebiotics: the concept revisited. J Nutr 2007;137:830-7.

5. Sorokulova I, Pinchuk I, Denayrolles M, et al. The safety of two Bacillus probiotic strains for human use. Dig Dis Sci 2008;53:954-63.

6. De Vecchi E, Drago L. Lactobacillus sporogenes or Bacillus coagulans. Misidentification or mislabeling? Int $\mathrm{J}$ Probio Prebio 2006;1:3-10.
7. Mohan JC, Arora R, Khalilullah M. Short term hypolipidemic effects of oral Lactobacillus sporogenes therapy in patients with primary dyslipidemias. Indian Heart J 1990;42:361-4.

8. Panda AK, Ramarao SV, Raju MVLN, Sharma SR. Dietary supplementation of probiotic Lactobacillus sporogenes on performance and serum biochemico-lipid profile of broiler chickens. J Poult Sci 2006;43: 235-40.

9. Abhari KH, Shekarforoush SS, Sajedianfard J, et al. The effects of prebiotic, probiotic and synbiotic diets including B. coagulans and inulin on rat intestinal microbiota. Iran J Vet Res 2015 [In Press].

10. Russell BL, Jelley SAA,Yousten AA. Selective medium for mosquito pathogenic strains of Bacillus sphaericus 2362. J Appl Environ Microbiol 1989;55:294-7.

11. Arjmandi BH, Craig J, Nathani S, Reeves RD. Soluble dietary fiber and cholesterol influence in vivo hepatic and intestinal cholesterol biosynthesis in rats. J Nut 1992;122:1559-65.

12. Kim M, Shin HK. The water-soluble extract of chicory influences serum and liver lipid concentrations, cecal short chain fatty acid concentrations and fecal lipid excretion in rats. J Nut 1998;128:1731-6.

13. Causey JL, Feirtag JM, Gallaher DD, et al. Effects of dietary inulin on serum lipids, blood glucose and the gastrointestinal environment in hypercholesterolemic men. Nutr Res 2000;20:191-201.

14. Letexier D, Diraison F, Beylot M. Addition of inulin to a moderately high-carbohydrate diet reduces hepatic lipogenesis and plasma triacylglycerol concentrations in humans. Am J Clin Nutr 2003;77:559-64.

15. Brighenti F, Casiraghi MC, Canzi E, Ferrari A. Effect of consumption of a readyto-eat breakfast cereal containing inulin on the intestinal milieu and blood lipids in healthy male volunteers. Eur J Clin Nutr 1999;53:726-33.

16. Hatakka K, Mutanen M, Holma R, et al. Lactobacillus rhamnosus LC705 together with Propionibacterium freudenreichii ssp shermanii JS administered in capsules is ineffective in lowering serum lipids. J Am Coll Nutr 2008;27:441-7.

17. Simons LA, Amansec SG, Conway P. Effect of Lactobacillus fermentumon serum lipids in subjects with elevated serum cholesterol. Nutr Metabolism Cardiovas Dis 2006;16:531-5.

18. Lewis SJ, Burmeister SA. Double-blind placebo-controlled study of the effects of Lactobacillus acidophilus on plasma lipids. Eur J Clin Nutr 2005;59:776-80.

19. Begley M, Hill C, Gahan CGM. Bile salt hydrolase activity in probiotics. Appl Environ Microbiol 2006;72:1729-38.

20. Ooi LG, Liong MT. Cholesterol-lowering effects of probiotics and prebiotics: a review of in vivo and in vitro findings. Int J Mol Sci 2010;11:2499-522.

21. Pereira DA, Gibson GR. Effects of consumption of probiotics and prebiotics on serum lipid levels in humans. Cri Rev Biochem Mol Biol 2002;37:259-81.

22. Kumar M, Nagpal R, Kumar R, et al. Cholesterol-lowering probiotics as potential biotherapeutics for metabolic diseases. Exp Diabetes Res 2012;2012:12-4. 